\title{
Retrospective study of pharyngeal pouch surgical management
}

* Pharyngeal pouches are uncommon (2/100000 per year) associated with older age.

* The most common primary treatment for pharyngeal pouches is endoscopic stapling.

* However, there are other potential treatment options such as open excision with cricopharyngeal myotomy that although can have greater potential risk, may confer better long term outcomes. Other options include laser division of cricopharyngeal bar.

\section{Patients and surgical treatment}

* 44 surgical procedures to treat pharyngeal pouch.

* 8 notes letters unavailable.

* $\quad \mathrm{N}=36$ included

\section{Fig 1: Age of patients}

\begin{tabular}{|l|l|}
\hline Age range & Patients \\
\hline$<50$ & $1(2.7 \%)$ \\
\hline $50-59$ & $6(16.7 \%)$ \\
\hline $60-69$ & $8(22.2 \%)$ \\
\hline $70-79$ & $9(25 \%)$ \\
\hline $80-89$ & $10(27.8 \%)$ \\
\hline $90-99$ & $2(5.6 \%)$ \\
\hline
\end{tabular}

Fig 2: Stage of pouch (Van Overbeek)

Patients

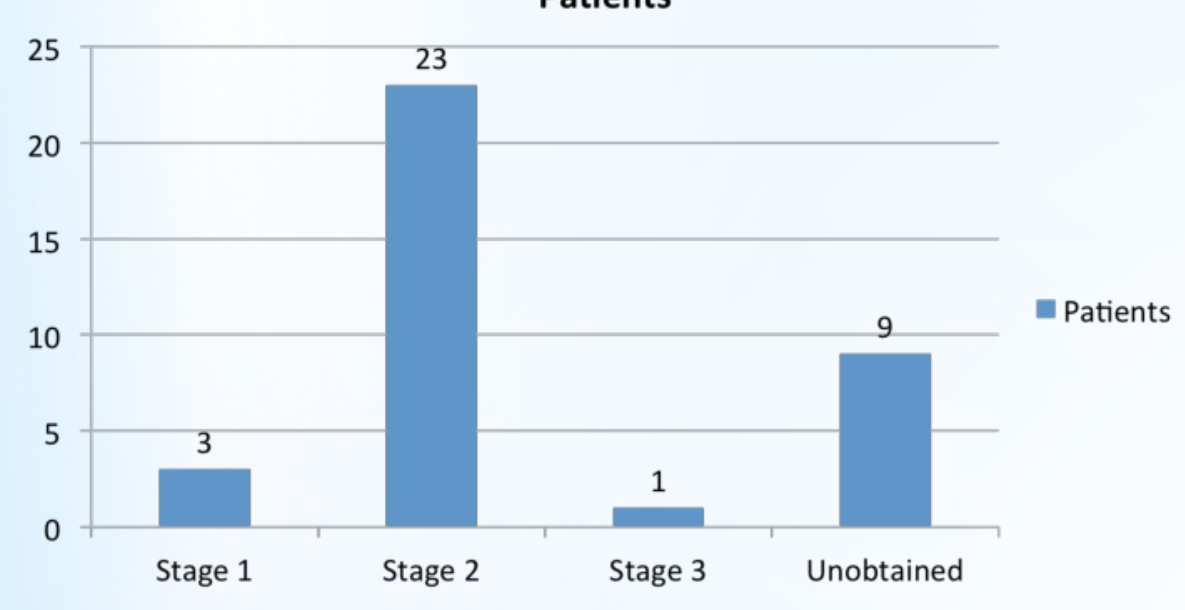

Fig 3: Surgical treatment

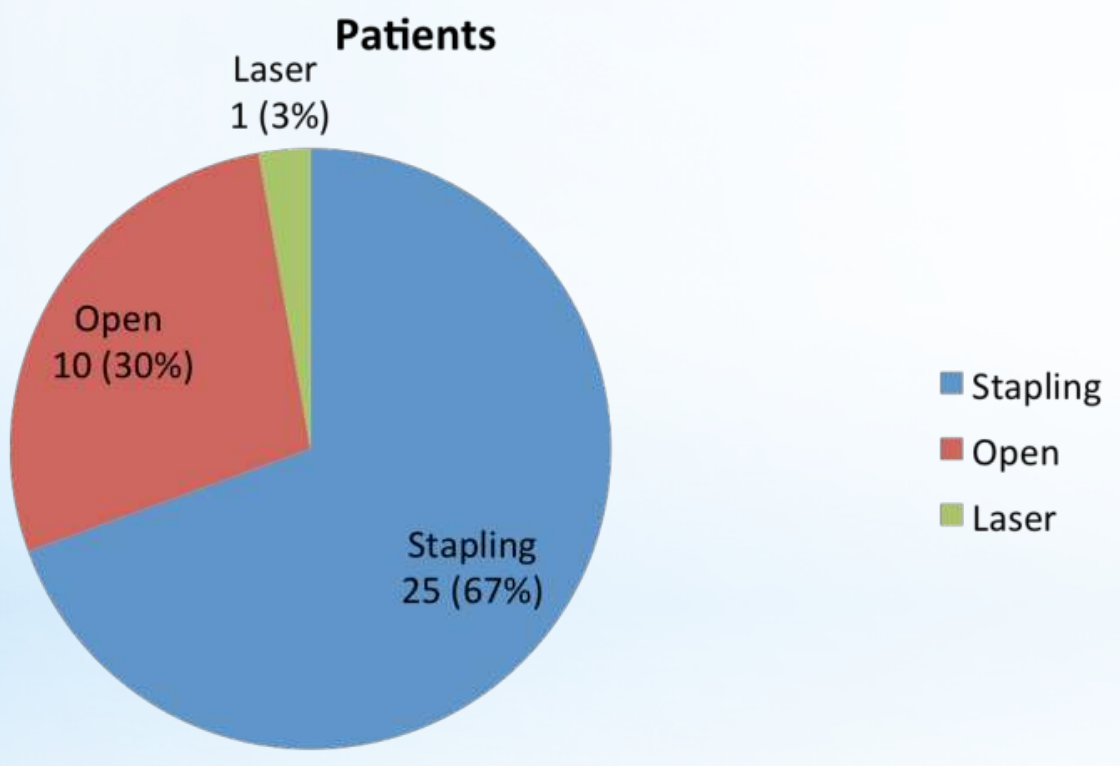

Open excision of pouch + cricopharyngeal myotomy $(\mathrm{N}=10)$

* Factors deciding surgery: - failed stapling, pouch too small, relatively young age- better long term outcome

* $10(100 \%)$ success

* 7 discharged after $1^{\text {st }}$ follow up

* 1 after second follow up (had post op infection)

* 1 ongoing follow up due to persistent globus

* Complications:

1 leak- treated with NG feed, same patient developed granulations on surgical wound

1 post op wound infection -IV abx

\section{Laser $(\mathbf{N}=1)$}

* Factors deciding surgery: recurrent pouch following open excision but difficult access

* Temporary improvement- required revision stapling

\section{Conclusion}

- High success rate of open surgery with better long term outcomes

\section{- Complication rates comparable to other studies}

\section{References}

* Zbären, P., et al. "Surgical treatment of Zenker's diverticulum: transcutaneous diverticulectomy versus microendoscopic myotomy of the cricopharyngeal muscle with CO2 laser." Otolaryngology--Head and Neck Surgery 121.4 (1999): 482-487.

* Laing, M. R., et al. "Surgery for pharyngeal pouch: audit of management with short-and long-term follow-up." Journal of the Royal College of Surgeons of Edinburgh 40.5 (1995): 315-318.

identification of diverticulum difficult

?more potential risk - infection,

haematoma, fistula, mediastinitis, leak, VC palsy

- Longer anaesthetic and hospital stay
* Feeley, Marc A., et al. "Zenker's diverticulum: analysis of surgical complications from diverticulectomy and cricopharyngeal myotomy." The Laryngoscope 109.6 (1999): 858-861. 